\title{
Repensando el proceso de enseñanza-aprendizaje de la morfología en el mundo post-pandemia
}

\author{
Rethinking the teaching-learning process of morphology in the post-pandemic world.
}

Francisco Garrido 1,2, Ana Barriga ${ }^{3}$, Ana María Leiva-Ordóñez ${ }^{3}$, Óscar Inzunza4

\section{Resumen}

Las ciencias morfológicas incluyen el estudio de tres disciplinas que analizan el cuerpo humano: la anatomía macroscópica, la histología y la embriología, las cuales forman parte central del ciclo básico en todas las carreras de la salud. El aprendizaje morfológico ocurre en el laboratorio y se basa en el estudio de disecciones cadavéricas, preparaciones histológicas o muestras del desarrollo embrionario, con el propósito de lograr una adecuada compresión de las características morfoestructurales del cuerpo humano. Sin embargo, la pandemia por SARS-CoV-2 significó un cambio profundo en la forma de enseñar, incorporando métodos de enseñanza-aprendizaje utilizados durante la pandemia que incluyen estrategias como los microscopios virtuales, atlas interactivos o videos de disecciones, lo que ha llevado a algunos a cuestionar la necesidad de utilizar los laboratorios de morfología. La educación virtual ha aparecido como una alternativa factible de mantener post-pandemia, sin embargo, el paso por el laboratorio es irreemplazable en la formación de profesionales de la salud, permitiendo el desarrollo de otras competencias más allá de la aplicación del conocimiento. La morfología seguirá siendo la piedra angular en la formación de profesionales de la salud. El desafío es como a través de la innovación y la creatividad incorporamos las nuevas tecnologías digitales, agregando un nuevo valor al estudio de esta disciplina.

Palabras clave: anatomía e histología; desarrollo humano; educación médica de pregrado; enseñanza; COVID-19.

\begin{abstract}
Morphological sciences include the study of three disciplines that analyze the human body: macroscopic anatomy, histology, and embryology, which are a central part of the basic cycle in all health careers. Morphological learning occurs in the laboratory, it is based on the study of cadaveric dissections, histological preparations, or samples of embryonic development to achieve an adequate compression of the morphological structure of the human body. The SARS-CoV-2 pandemic meant a profound change in the way of teaching. These, teaching-learning methods that have been implemented during the pandemic include virtual microscopes, interactive atlases, or videos of dissections, which has led some teachers to question the usefulness of morphology laboratories. Virtual education has appeared as a feasible alternative to maintaining in post-pandemic; however, going through the laboratory is irreplaceable in the training of health professionals, allowing the development of other skills beyond the application of knowledge. The morphology will continue to be the cornerstone in the education of health professionals. The challenge is how through innovation and creativity, we incorporate new digital technologies, adding a new value to the study of this discipline.
\end{abstract}

Keywords: anatomy and histology; human development; undergraduate medical education; teaching; COVID-19.

Fecha de envío: 2021-11-10 - Fecha de aceptación: 2021-12-21

\section{Introducción}

Las ciencias morfológicas incluyen el estudio de tres disciplinas que abordan el estudio del cuerpo humano: la anatomía macroscópica, la histología o anatomía microscópica y la embriología o biología del desarrollo. En el modelo flexneriano estas disciplinas forman parte central del ciclo de ciencias básicas de la formación de todos los profesionales del área de la salud, las cuales son abordadas en función de las competencias definidas según el perfil profesional,

(1) Departamento de Radiología, Escuela de Medicina, Pontificia Universidad Católica de Chile. Santiago, Chile.

(2) Centro de Educación Médica y Ciencias de la Salud, Facultad de Medicina, Pontificia Universidad Católica de Chile. Santiago, Chile.

(3) Instituto de Anatomía, Histología y Patología, Facultad de Medicina, Universidad Austral de Chile. Valdivia, Chile.

(4) Departamento de Anatomía Normal, Escuela de Medicina, Pontificia Universidad Católica de Chile. Santiago, Chile.

Autor de correspondencia: aleiva@uach.cl

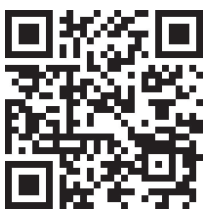


con diferentes grados de profundidad y organizadas en cursos independientes o en asignaturas integradas, con enfoques sistemáticos (por sistemas) o topográficos (regionales o por segmentos corporales) (Louw et al., 2019). En dichas asignaturas, la mayor parte del tiempo curricular se dedica a sesiones teóricas, lo cual es complementado con la revisión de bibliografía clásica y el trabajo práctico en el laboratorio (Rodríguez et al., 2015).

A pesar de que las ciencias morfológicas son consideradas un pilar fundamental en la formación de un profesional de la salud, la necesidad de incorporar nuevas competencias y habilidades en la formación de pregrado ha traído como consecuencia una significativa reducción de las horas lectivas dedicadas a los cursos morfológicos (Inzunza, 2014, Kuhlmann et al., 2021). Dichos cambios han demandado enormes esfuerzos por parte de los académicos por reducir contenidos sin sacrificar la calidad de la formación (Inzunza et al., 2007; Rodríguez-Herrera et al., 2019; Garrido et al., 2020). Paradójicamente, esta disminución del creditaje de los cursos de morfología se ha dado en paralelo con los avances de las imágenes médicas, cuya interpretación demanda un elevado conocimiento anatómico, lo que viene a reforzar el hecho que las ciencias morfológicas sean consideradas un pilar fundamental en la formación de un profesional de la salud (Inzunza et al., 2007; Kuhlmann et al., 2021).

La pandemia por SARS-CoV-2 originada a fines del año 2019, ha significado enormes desafíos que han alcanzado dimensiones globales no solo en el ámbito sanitario, sino que además ha impactado profundamente en lo económico, social, político y educativo. A nivel formativo universitario, hemos sido testigos del cierre de todas las escuelas y campus, migrando obligada e inesperadamente a una educación basada en plataformas virtuales. Este nuevo escenario de educación remota es visto por muchos como una oportunidad que vino a revolucionar los procesos educativos, posicionando a la educación virtual como una alternativa interesante y factible de mantener en el mundo post-pandemia, lo que trae como desafío a los educadores en ciencias de la salud la necesidad de repensar y agregar valor a los encuentros presenciales con nuestros estudiantes (Gibbs, 2020). La tabla 1 resume algunas oportunidades y desafíos reportados en la literatura reciente para la educación en ciencias de la salud.

Tabla 1: Desafíos y oportunidades en la educación en ciencias de la salud en tiempos de pandemia (De Carvalho et al., 2021; Hayat et al., 2021; Khasawneh et al., 2021; Kuhlmann et al., 2021)

\begin{tabular}{l|l}
\hline Desafíos & Oportunidades \\
\hline Alfabetización virtual asegurando un nivel mínimo de habilidades. & Apertura hacia la modalidad b-learning. \\
\hline Asegurar la calidad del componente virtual del b-learning. & Aumento de la utilidad percibida por parte de los usuarios. \\
\hline Promoción de aprendizajes significativos. & Creación de entornos educativos flexibles y enriquecidos. \\
\hline Potenciar trabajo en red y las colaboraciones interdisciplinarias. & Colaboración multicéntrica. \\
\hline Mejorar las brechas en el acceso a la conectividad y dispositivos. & Invertir en infraestructura orientada al desarrollo del $b$-learning. \\
\hline Priorizar contenidos. & Optimizar los tiempos de enseñanza. \\
\hline Ofertas educativas sin límite geográfico. & Permitir la educación sincrónica en sitios remotos. \\
\hline
\end{tabular}

Otro de los desafíos importantes a tener presente en este nuevo escenario, es la planificación de la docencia con un enfoque centrado en el estudiante y de qué forma la docencia universitaria responde a las características de los estudiantes de hoy. Como parte de su modelo SPICES, Ronald Harden define la docencia centrada en el estudiante como un enfoque en el cual el alumno es protagonista de su propio aprendizaje, decidiendo sus propios objetivos de aprendizaje y seleccionando los recursos educativos apropiados para lograr esos objetivos. Además, promueve la motivación y permite preparar a los estudiantes para la educación continua (Harden et al., 1984). Recientemente, en una propuesta de actualización del modelo SPICES, Olle ten Cate incluye los recursos educativos digitales como parte de la "educación médica moderna" (ten Cate, 2014), lo que es coherente con la forma de aprender de los estudiantes de hoy, que al ser nativos digitales valoran de manera importante la conectividad. Ambos aspectos, fondo y forma, son muy relevantes para todo proceso educativo en las carreras de la salud en general y constituyen una oportunidad para la innovación en la educación en las ciencias morfológicas.

El propósito del presente artículo es plantear algunas reflexiones sobre el futuro del proceso de enseñanza y aprendizaje que impondrá el escenario post-pandemia en la formación morfológica de los estudiantes del área de la salud.

\section{La educación morfológica en tiempos de COVID-19}

Tradicionalmente la enseñanza de las ciencias morfológicas se centra en un acercamiento in situ al objeto de estudio, con el propósito de lograr una adecuada compresión de las características morfoestructurales del cuerpo humano, siendo el laboratorio el lugar por excelencia donde ocurre este proceso de enseñanza-aprendizaje: ya sea frente al cadáver, un microscopio o muestras de las diferentes 
etapas del desarrollo embrionario. Estas actividades permiten al estudiante confirmar empíricamente la situación de órganos, tejidos y sistemas corporales, y su correlación clínica (Inzunza, 2008); proceso que es apoyado por otros elementos tan sencillos y disponibles como la tiza y el pizarrón, pasando por las láminas anatómicas, atlas anatómicos e histológicos, diapositivas o videos, hasta softwares de disección desarrollados con alta tecnología y realidad virtual (Cárdenas-Valenzuela, 2019).

La suspensión de las actividades presenciales en todos los niveles educativos a contar de marzo del año 2020, a consecuencia de la pandemia por COVID-19, significó una tensión para estudiantes y profesores. La docencia en morfología se ha sustentado en el uso de recursos virtuales, con clases teóricas sincrónicas y asincrónicas preparadas por académicos que en su mayoría no estaban capacitados para entregar una docencia virtual efectiva. Los docentes describen esta situación como un cambio brusco que inicialmente generó ansiedad e incertidumbre, pero que se transformó en una experiencia novedosa, enriquecedora y llena de aprendizaje (Kuhlmann et al., 2021).

Con la pandemia, no solo se limitó completamente el acceso a la disección de material cadavérico, recurso metodológico por excelencia para acercarse a la morfología, sino que también a modelos, especímenes de museo, huesos, muestras histológicas, entre otros elementos, a los cuales los estudiantes tienen acceso en los laboratorios y que apoyan la compresión de cuerpo humano (Singal et al., 2020).

La imposibilidad de utilizar los laboratorios de morfología obligó a buscar nuevos métodos de enseñanza-aprendizaje, reportándose en diversas publicaciones, estrategias novedosas que incluyen entre otras, el uso de microscopios virtuales, atlas interactivos y videos en base a disecciones, entre otros. A modo de ejemplo, Martin (2021) reporta el uso de la aplicación Complete 3-D Anatomy, como complemento al aprendizaje de la anatomía humana dentro del formato de las sesiones sincrónicas vía Zoom. Los estudiantes señalaron que la aplicación era un complemento útil para la comprensión de la anatomía y, por otro lado, los estudiantes que la utilizaron obtuvieron un mayor porcentaje de aprobación del curso en comparación con el grupo control. Por otro lado, Harret et al. (2021) diseñaron laboratorios virtuales de anatomía, que incluyó videos de disecciones en pabellón, lo que buscaba preservar los beneficios de la experiencia de disección para los estudiantes de primer año, en particular de la carrera de medicina. Las evaluaciones de los estudiantes de las sesiones en línea y de los cursos en general, fueron altamente positivas. Finalmente, para histología y embriología, Rojas et al. (2020) presentan una experiencia con el microscopio virtual, con el fin de poner a disposición de los estudiantes y docentes el material de las actividades prácticas en todo momento y lugar, lo que resultó bastante beneficioso, otorgando al estudiante la libertad de observar y describir las estructuras.

Aunque es muy pronto para dimensionar si estos recursos digitales son suficientes para adquirir las competencias requeridas, existen opiniones divididas. En particular en lo que a la enseñanza de la anatomía se refiere, hay quienes sostienen que "por ahora, no hay nada que reemplace la experiencia única de la disección en el cadáver" (Hayat, 2021). La posibilidad de contar regularmente con material cadavérico para la docencia morfológica ofrece a los estudiantes de las carreras de la salud la oportunidad de comprobar, de primera mano, las características y situación de los órganos de los distintos sistemas corporales y su relación con la enfermedad y la muerte, por lo que la imposibilidad de experimentar esta experiencia, impone una brecha y dificultad para la comprensión de los temas que requieren la práctica y orientación visual (Khasawneh, 2021). Además, en la exploración anatómica es posible adquirir la conceptualización tridimensional de la topografía de las distintas regiones corporales y la relación recíproca de las estructuras incluidas en ellas. Las características de las diferentes estructuras anatómicas en términos de color, textura, consistencia, situación y resistencia sólo pueden ser verificadas e internalizadas en el trabajo con material cadavérico. Incluso, para muchos el laboratorio de anatomía es considerado un "taller" donde se realizan e inculcan virtudes como la confiabilidad, empatía, o compasión (Pearson, 2020). Es a partir de dicha experiencia, que los estudiantes de las carreras de la salud tienen la oportunidad única de conocer a su primer paciente en este laboratorio; encuentro que define la realidad de la vida y proyecta al estudiante a su objetivo final: cuidar la salud del paciente vivo (Inzunza, 2008).

\section{Imaginando el laboratorio de morfología del futuro}

Contrariamente a lo expuesto, hay quienes cuestionan la necesidad de continuar utilizando los pabellones de disección, reemplazándolo por recursos tecnológicos que permitan potenciar el aprendizaje de los estudiantes (McMenamin et al., 2018; Singal et al., 2020). La mantención de laboratorios de anatomía humana requiere una inversión importante de recursos financieros por parte de las instituciones de educación superior, los que no solo se destinan a la contratación de docentes y personal de apoyo calificado, sino que también requiere de equipamiento especializado para el tratamiento y mantención del material cadavérico de acuerdo con los estándares de bioseguridad que exige la legislación vigente.

Es indiscutible que las tecnologías de la información han proporcionado recursos metodológicos alternativos a la disección cadavérica como los videos, atlas digitales, impresiones 3D, realidad aumentada 
o realidad virtual, incluso mucho antes de iniciada la pandemia (Iwanaga et al., 2021). Previo a la pandemia, se ha reportado que la autopercepción de aprendizaje y satisfacción de los estudiantes utilizando los recursos digitales es menor, comparado con los programas de morfología tradicionales que utilizan la disección (Mathiowetz et al., 2016). Los estudiantes consideran que el trabajo con material cadavérico es parte crucial de su desarrollo como futuro profesional de la salud, considerándolo no sólo un rito dentro de su paso por la universidad, sino el método más efectivo para la adquisición de una amplia gama de habilidades (Franchi, 2020).

Autores como de Carvalho et al. (2021), plantean que el laboratorio de morfología del futuro debe transformarse en un "estudio de anatomía", que invite a estudiantes y profesores a ser co-creadores capaces de desarrollar contenido en vivo. Para ello se requiere de equipamiento audiovisual apropiado que permita explorar el cuerpo humano desde diferentes puntos de vista, con aplicación clínica, como las imágenes médicas, las muestras de patología o desde la cámara de laparoscopía de un pabellón de operaciones. En este nuevo escenario para el estudio de morfología, los estudiantes podrán explorar la anatomía humana con perspectivas diferentes: cámaras capturando diversos ángulos, magnificaciones de determinados segmentos o la posibilidad de visualizar áreas difíciles de explorar en la docencia tradicional basada en la disección (de Carvalho et al., 2021).

Sin embargo, a pesar de los avances de las tecnologías, el centro de la docencia morfológica seguirá siendo el cuerpo humano, lo que justifica y pone en evidencia la necesidad de mantener y potenciar los programas de donación de cuerpos a la ciencia y la docencia. En nuestro medio nacional, son pocas las instituciones que cuentan con un programa de donación de cuerpos para la docencia morfológica, contándose entre ellas a la Universidad de Antofagasta, Universidad de Santiago, Universidad Católica del Maule, Universidad de Concepción, Universidad de la Frontera, Universidad Austral de Chile, Universidad de los Andes, Universidad de Chile y la Pontificia Universidad Católica de Chile, de un total de más de 40 universidades que dictan carreras de la salud en el país; siendo los programas de las dos últimas instituciones mencionadas las que tienen más de dos décadas de historia. Este dato es importante, ya que el curso temporal de los programas de donación desde el momento que se inician hasta que ya están en régimen de recepción regular de donantes es de al menos 10 años. Lamentablemente, la pandemia por SARS-CoV-2 ha afectado al desarrollo y continuidad de estos programas, existiendo varias instituciones que actualmente no están recibiendo cadáveres de donantes.

Otro elemento importante de este laboratorio morfológico del futuro son las imágenes médicas. La utilización de las imágenes médicas como recurso de apoyo al aprendizaje de la anatomía data de la década de 1930, lo que sucedió varios años después de su descubrimiento por parte del físico Wilhelm Conrad Röntgen en Alemania. Posteriormente, se popularizó la incorporación de negatoscopios en los pabellones de disección, que permitieron complementar lo estudiado en los pasos prácticos con placas radiográficas (Cárdenas-Valenzuela, 2019). A lo largo del tiempo, otras metodologías se han incorporado para cumplir con el mismo objetivo: presentaciones de anatomía radiológica en el laboratorio de disección, autoinstrucción con atlas o recursos generados por el equipo docente, disecciones virtuales con tomografía computada o resonancia magnética, correlación con demostración a través de estudios ecográficos, entre otros (Phillips et al., 2013). Es por ello por lo que los departamentos de anatomía y radiología se potencian mutuamente en este tipo de actividades. Por una parte, los radiólogos introducen los conceptos básicos de imagenología precozmente durante la formación de pregrado, promueven y fortalecen su propio desarrollo académico y, por otro lado, los morfólogos se benefician al poder demostrar a sus estudiantes la aplicación clínica de los contenidos incluidos en sus cursos, avanzando en la comprensión precoz del lenguaje utilizado en la medicina (Chowdhury et al., 2008). En este sentido, algunos autores proponen que la mitad del tiempo curricular del curso de anatomía debería estar destinado a la anatomía con aplicación radiológica (Caswell et al., 2015). Existen varios reportes en la literatura educacional que demuestran que el uso de las imágenes médicas como recurso de enseñanza-aprendizaje se traduce una mejoría en la satisfacción de los estudiantes y en el rendimiento en las evaluaciones del curso de anatomía (Phillips et al, 2012; Webb \& Choi, 2014; Chew et al., 2020). Los radiólogos son los anatomistas del futuro (Pathiraja et al., 2014) y la radiología puede ser considerada, por lo tanto, como la cuarta ciencia morfológica.

En el contexto del currículo de pregrado, la anatomía debe estar al servicio de la clínica; los detalles anatómicos se hacen relevantes con la adecuada orientación y aplicación práctica del conocimiento anatómico. Históricamente, los avances tecnológicos han impuesto una nueva forma de"ver"la anatomía; a inicios del siglo XX fue la anatomía radiológica, después la anatomía seccional y más recientemente la anatomía endoscópica y la anatomía laparoscópica. No sabemos que nos depararán los nuevos desarrollos de exploración médica, sin embargo, es muy probable que estén presentes en el laboratorio de morfología del futuro.

\section{Conclusiones}

La formación en el área de las ciencias morfológicas impone numerosos desafíos para el futuro. Su importancia es central en la educación de las profesiones de la salud, por lo que es necesario que la innovación y la creatividad en los procesos de enseñanza - aprendizaje entreguen un 
nuevo valor al estudio de la disciplina. A pesar de haber incorporado nuevas tecnologías y recursos educativos, como las imágenes médicas, que complementan y contextualizan lo aprendido con la clínica, el cadáver es un recurso único, con una historia que trae implícita la generosidad de la persona que altruistamente donó su cuerpo a la ciencia para contribuir a la formación de los futuros profesionales del área de la salud. La frase del profesor José Joaquín Aguirre Campos (1822 - 1901), destacado anatomista y rector de la Universidad de Chile, se mantiene plenamente vigente hasta nuestros días: "vamos a estudiar la Anatomía, el cadáver será nuestro maestro; los libros nuestra guía, y yo los ayudaré en el estudio" (Cárdenas-Valenzuela, 2020). Estamos ante un cambio de paradigma en la formación de los profesionales de la salud. Las lecciones aprendidas en este período de pandemia COVID-19 constituyen una oportunidad para potenciar estrategias de aprendizaje que logren combinar y optimizar métodos tradicionales de enseñanza-aprendizaje con las nuevas tecnologías.

\section{Referencias}

Cárdenas-Valenzuela J. (2019). Enseñanza de la Anatomía. Uso de Medios en el Aula. International Journal of Morphology 37, 1123-1129.

Cárdenas-Valenzuela J. (2020). Historia de la Anatomía en Chile Parte II. El Alma Mater. International Journal of Morphology 38, 1074-1089.

Caswell FR., Venkatesh A. \& Denison A. (2015). Twelve tips for enhancing anatomy teaching and learning using radiology. Medical Teacher 37, 1067-1071.

Chew C, O'Dwyer P, Young D. \& Gracie J. (2020). Radiology teaching improves Anatomy scores for medical students. British Journal of Radiology 93, 20200463.

Chowdhury R., Wilson I. \& Oeppen R. (2008). The departments of radiology and anatomy: new symbiotic relations?. Clinical Radiology 63, 918-920.

De Carvalho M, Hafferty F. \& Pawlina W. (2021). Anatomy 3.0: Rediscovering Theatrum Anatomicum in the wake of Covid-19. Anatomical Sciences Education 14, 528-535.

Franchi T. (2020). The Impact of the Covid-19 Pandemic on Current Anatomy Education and Future Careers: A Student's Perspective. Anatomical Sciences Education 13, 312-315.

Garrido F, LabbéT, Paris E. \& Ríos J. (2020). Formación científica en el pregrado de medicina en Chile: ¿dónde estamos? y ¿hacia dónde vamos? Revista Médica de Chile 148, 1011-1017.

Gibbs T. (2020). The Covid-19 pandemic: Provoking thought and encouraging change. Medical Teacher 42, 738-740.
Harden R, Sowden S. \& Dunn W. (1984). Educational strategies in curriculum development: the SPICES model. Medical Education 18, 284-297.

Harret K, McGinn M, Edwards C, Warren Foster K. \& Meredith M. (2021). Crashing from cadaver to computer: Covid-driven crisis-mode pedagogy spawns active online substitute for teaching gross anatomy. Anatomical Sciences Education 14, 536-551.

Hayat A, Keshavarzi M, Zare S, Bazrafcan L, Rezaee R, Faghihi S, Amini A. \& Kojuri, J. (2021). Challenges and opportunities from the COVID-19 pandemic in medical education: a qualitative study. BMC Medical Education 21, 247.

Inzunza O. (2008). Competencias Generales en Medicina, Rol de la Anatomía. International Journal of Morphology 26, 243-246.

Inzunza O. (2014). Morfología, los Nuevos Desafíos para el 2015. International Journal of Morphology 32, 789-793.

Inzunza O, Vargas A. \& Bravo H. (2007). Anatomy and neuroanatomy the most impair in the curricular reform. International Journal of Morphology 25, 825-830.

Iwanaga J, Loukas M, Dumont A. \& Tubbs R. (2021). A review of anatomy education during and after the COVID-19 pandemic: Revisiting traditional and modern methods to achieve future innovation. Clinical Anatomy 34, 108-114.

Khasawneh R. (2021). Anatomy Education of Medical Students During the COVID 19 Pandemic. International Journal of Morphology 39,1264-1269.

Kuhlmann A, Guillén J, Cortés M, Gutiérrez M, Alvarado P, Sánchez M, García A, Mera C, González L. \& Franco J. (2021). Perception of Teachers in the Department of Morphological Sciences Regarding the Change from Classroom-Based Teaching to Remote Modality, in the Context of the Crisis Due to the COVID-19 Pandemic. Universitas Medica 62, e31774.

Louw G, Eizenberg N. \& Carmichael S. (2009). The place of anatomy in medical education: AMEE Guide no 41. Medical Teacher 31, 373-386.

Mathiowetz V, Yu C. \& Quake-Rapp C. (2016). Comparison of a gross anatomy laboratory to online anatomy software for teaching anatomy. Anatomical Sciences Education 9, 52-59.

McMenamin P, McLachlan J, Wilson A., McBride J., Pickering J., Evans D. \& Winkelmann A. (2018). Do we really need cadavers anymore to learn anatomy in undergraduate education?. Medical Teacher 40, 1020-1029. 
Pathiraja F., Little D. \& Denison A. (2014). Are radiologists the contemporary anatomists?. Clinical Radiology 69, 458-461.

Pearson S. (2020). Anatomy: Beyond the COVID-19 Pandemic. Academic Medicine 95, e1.

Phillips A, Smith S. \& Straus C. (2013). The Role of Radiology in Preclinical Anatomy. A Critical Review of the Past, Present, and Future. Academic Radiology 20, 297-304.

Phillips A, Smith S, Ross C. \& Straus C. (2012). Improved Understanding of Human Anatomy through Self-guided Radiological Anatomy Modules. Academic Radiology 19, 902-907.

Rodríguez R., Guevara R., Urrutia M, González-Villalva A. \& Fortoul T. (2015). La enseñanza de las ciencias básicas en medicina. En Educación Médica Teoría y Práctica, ed. Sánchez M., Lifshitz A., Vilar P., Martínez A., Varela M. \& Graue E, pp. 215 - 219. Editorial Elsevier. España.

Rodríguez-Herrera R, Losardo RJ. \& Binvignat O. (2019). Human anatomy an essential discipline for patient safety. International Journal of Morphology 37, 241-250.
Rojas M, Cuevas F, Smok C, Roa I, Conei D, Prieto R. \& del Sol M. (2020). ¡Estudiando el Desarrollo Embrionario y Fetal con el Microscopio Virtual! En Tiempos de Covid-19. International Journal of Morphology 38, 1296-1301.

Rosario M. (2021). Gross Anatomy during COVID-19: The Effectiveness of Utilizing a 3-D Anatomy Application among Occupational Therapy Students in a Pandemic-induced Online Course. Journal of Learning and Teaching in Digital Age 6, 90-96.

Singal A, Bansal A. \& Chaudhary P. (2020). Cadaverless anatomy: Darkness in the times of pandemic Covid-19. Morphologie 104, 147-150.

Ten Cate O. (2014). Thirty years after SPICES - is it time for SPICES 2.0? Accedido en https://www.mededworld.org/hardens-blog/ reflection-items/September-2014/Thirty-years-after-SPICES-is-ittime-for-SPICES-2.aspx el 27 de noviembre de 2021.

Webb A. \& Choi S. (2014). Interactive radiological anatomy eLearning solution for first year medical students: Development, integration, and impact on learning. Anatomical Sciences Education 7, 350-360. 